\title{
Definition of a COPD self-management intervention: International Expert Group consensus
}

\author{
Tanja W. Effing ${ }^{1,2}$, Jan H. Vercoulen ${ }^{3}$, Jean Bourbeau ${ }^{4}$, Jaap Trappenburg ${ }^{5}$, \\ Anke Lenferink ${ }^{6}$, Paul Cafarella ${ }^{1,2}$, David Coultas ${ }^{7}$, Paula Meek $^{8}$, \\ Paul van der Valk ${ }^{6}$, Erik W.M.A. Bischoff ${ }^{9}$, Christine Bucknall ${ }^{10}$, \\ Naresh A. Dewan ${ }^{11}$, Frances Early ${ }^{12}$, Vincent Fan ${ }^{13,14}$, Peter Frith ${ }^{1,2}$, \\ Daisy J.A. Janssen ${ }^{15}$, Katy Mitchell ${ }^{16}$, Mike Morgan ${ }^{17}$, Linda Nici ${ }^{18}$, Irem Patel ${ }^{19}$, \\ Haydn Walters ${ }^{20}$, Kathryn L. Rice ${ }^{21,22}$, Sally Singh ${ }^{16}$, Richard Zuwallack ${ }^{23}$, \\ Roberto Benzo ${ }^{24}$, Roger Goldstein ${ }^{25}$, Martyn R. Partridge ${ }^{26}$ and \\ Job van der Palen 6,27
}

ABSTRACT There is an urgent need for consensus on what defines a chronic obstructive pulmonary disease (COPD) self-management intervention. We aimed to obtain consensus regarding the conceptual definition of a COPD self-management intervention by engaging an international panel of COPD selfmanagement experts using Delphi technique features and an additional group meeting.

In each consensus round the experts were asked to provide feedback on the proposed definition and to score their level of agreement ( $1=$ totally disagree; $5=$ totally agree). The information provided was used to modify the definition for the next consensus round. Thematic analysis was used for free text responses and descriptive statistics were used for agreement scores.

In total, 28 experts participated. The consensus round response rate varied randomly over the five rounds (ranging from $48 \%(n=13)$ to $85 \%(n=23)$ ), and mean definition agreement scores increased from 3.8 (round 1) to 4.8 (round 5) with an increasing percentage of experts allocating the highest score of 5 (round 1: $14 \%(\mathrm{n}=3)$; round 5: $83 \%(\mathrm{n}=19)$ ).

In this study we reached consensus regarding a conceptual definition of what should be a COPD self-management intervention, clarifying the requisites for such an intervention. Operationalisation of this conceptual definition in the near future will be an essential next step.

@ERSpublications

Consensus of a conceptual definition of what should be a COPD self-management intervention with its requisites http://ow.ly/ZfroF

Received: Jan 062016 | Accepted after revision: March 032016 | First published online: May 122016

Support statement: This project was self-funded but at the time of the 2015 ERS International Congress in Amsterdam AstraZeneca paid the costs of a meeting room in a hotel and some refreshments to use for our deliberations but took no part in the meeting.

Conflict of interest: Disclosures can be found alongside the online version of this article at erj.ersjournals.com

The content of this work is not subject to copyright. Design and branding are copyright @ERS 2016. 
Affiliations: ${ }^{1}$ Dept of Respiratory Medicine, Southern Adelaide Local Health Network, Adelaide, Australia. ${ }^{2}$ School of Medicine, Flinders University, Adelaide, Australia. ${ }^{3}$ Dept of Medical Psychology and Dept of Pulmonary Diseases, Radboud University Medical Center, Nijmegen, The Netherlands. ${ }^{4}$ Respiratory Epidemiology and Clinical Research Unit, Research Institute of the McGill University Health Centre, McGill University, Montreal, QC, Canada. ${ }^{5}$ Dept of Rehabilitation, Nursing Science \& Sports, University Medical Center Utrecht, Utrecht, The Netherlands. ${ }^{6}$ Dept of Pulmonary Medicine, Medisch Spectrum Twente, Enschede, The Netherlands. ${ }^{7}$ VA Portland Health Care System and Dept of Medicine, Oregon Health \& Science University, Portland, OR, USA. ${ }^{8}$ University of Colorado College of Nursing, Aurora, CO, USA. ${ }^{9}$ Dept of Primary and Community Care, Radboud University Medical Center, Nijmegen, The Netherlands. ${ }^{10}$ Dept of Respiratory Medicine, Glasgow Royal Infirmary, Glasgow, UK. ${ }^{11}$ Division of Pulmonary Critical Care and Sleep Medicine, Creighton University and Omaha Veterans Affairs Medical Centre, Omaha, NE, USA. ${ }^{12}$ Centre for SelfManagement Support, Cambridge University Hospitals NHS Foundation Trust, Addenbrooke's Hospital, Cambridge, UK. ${ }^{13}$ VA Puget Sound Health Care System, Seattle, WA, USA. ${ }^{14}$ Dept of Medicine, University of Washington, Seattle, WA, USA. ${ }^{15}$ Dept of Research \& Education, CIRO, Centre of Expertise for Chronic Organ Failure, Horn, The Netherlands. ${ }^{16}$ Centre for Exercise and Rehabilitation Science, University Hospitals of Leicester, Leicester, UK. ${ }^{17}$ Dept of Respiratory Medicine, Glenfield Hospital, University Hospitals of Leicester NHS Trust, Leicester, UK. ${ }^{18}$ Dept of Pulmonary and Critical Care, Providence Veterans Administration Medical Center, Providence, RI, USA. ${ }^{19}$ Dept of Respiratory Medicine, King's College Hospital NHS Trust, London, UK. ${ }^{20} \mathrm{CRE}$ for Chronic Respiratory Disease, School of Medicine, University of Tasmania, Hobart, Australia. ${ }^{21}$ Pulmonary Section, Dept of Medicine, VA Medical Center, Minneapolis, MN, USA. ${ }^{22}$ Dept of Medicine, University of Minnesota, Minneapolis, MN, USA. ${ }^{23}$ Dept of Pulmonary and Critical Care, St. Francis Hospital and Medical Center, Hartford, CT, USA. ${ }^{24}$ Mindful Breathing Lab, Mayo Clinic, Rochester, MN, USA. ${ }^{25}$ West Park Healthcare, University of Toronto, Toronto, ON, Canada. ${ }^{26}$ National Heart and Lung Institute, Imperial College, London, UK. ${ }^{27}$ Dept of Research Methodology, Measurement and Data Analysis, University of Twente, Enschede, The Netherlands.

Correspondence: Tanja Effing, Dept of Respiratory Medicine, Repatriation General Hospital, Daws Road, Daw Park, SA 5041, Australia. E-mail: tanja.effingahealth.sa.gov.au

\section{Introduction}

Self-management interventions have previously been described as structured interventions for individuals, aimed at improving self-health behaviour, developing self-management skills and increasing the patient's responsibility for healthcare decisions [1-4]. Self-management skills are important in patients with chronic obstructive pulmonary disease (COPD) who are responsible for their day-to-day care [1]. A core feature of COPD is that symptoms may change frequently due to exacerbations of disease and disease progression [5]. The use of COPD action plans in COPD self-management interventions may support patients to respond to changing symptoms, and thereby make appropriate decisions regarding their self-management. The potential benefits of self-management in COPD have been reinforced by a recent Cochrane review [6], which demonstrated positive outcomes in hospital admissions and health-related quality of life.

Approaches that are currently considered as self-management interventions range from simply providing an information leaflet [7] to more complex interventions that include several educational sessions combined with various content, such as an exercise programme, optimisation of care plans, ongoing nurse support [8-10], or even a comprehensive multidisciplinary pulmonary rehabilitation programme [11]. This heterogeneity complicates the provision of recommendations with regard to the most effective content of COPD self-management interventions and limits the fine-tuning of COPD self-management interventions $[6,10,12]$. Since 2003, most definitions of self-management have involved slight alterations of the one formulated by BOURBEAu et al. [10]. In this definition, formalised education is centre stage but while COPD self-management interventions often include education and action plans, the intervention is considered to be more than the sum of these two components [13]. The focus of a COPD self-management intervention is sustained behavioural change, effected by incorporating those components that are effective in achieving this goal $[3,11]$.

In the operational definition used in the latest Cochrane review, "education" is considered as one of the COPD self-management intervention components [6] and the importance of behavioural change techniques is acknowledged to some extent by requiring an iterative process of interaction between patient and healthcare provider in the intervention. However, behavioural change techniques have not been considered as mandatory [6]. In addition, the definition states that at least two out of six listed self-management intervention components need to be part of the overall intervention [6]. However, these components differ slightly from those in a recent large individual patient data meta-analysis on COPD self-management interventions [12]. The differences in these operational definitions are not surprising as, to date, no consensus has been reached regarding the conceptual definition of COPD self-management.

Given the strong interest from health system administrators and funders in strategies that divert costs from expensive medical practitioners to unpaid patients and their informal carers, there is a strong need in both research and clinical settings for consensus on the nature and entity of a COPD self-management intervention. The current ambiguity regarding a definition for a COPD self-management intervention not 
only impacts on the ability to provide clear-cut advice regarding effective self-management interventions and their implementation in clinical practice, but also creates a confusing landscape for those wishing to develop and investigate the effectiveness of self-management interventions. To move forward we need consensus about what defines a COPD self-management intervention. Only then can we advance towards operationalisation of the definition. We aimed to obtain consensus regarding the conceptual definition of a COPD self-management intervention among an international panel of experts in the field of COPD self-management.

\section{Methods}

Experts who attended a self-management roundtable during the American Thoracic Society annual conference in May 2014 agreed to start a formal process to develop a definition for a COPD self-management intervention. This process included email iterative polling of experts for their opinions and feedback regarding a proposed definition (figure 1). The process would be continued until the group felt that the maximum feasible consensus was achieved. The length of the process was not pre-defined, but started in August 2014 and ended in August 2015.

The original expert group consisted of nine individuals who had been working together since 2007 with the aim of bringing greater clarity to the terminologies, content and processes within COPD self-management [3]. This group was extended with the addition of another 19 people who were identified as being highly involved in COPD self-management research and/or clinical work between 2007 and 2014. These individuals had expressed interest in being part of the expert group or were actively invited. Data were collected using features of a Delphi technique [14], which was complemented with an additional group meeting [15]. The exact methods are described below.

The process was led by one member of the original expert group (T.W. Effing). After each round the feedback was processed and discussed within a reference group of five experts (J.H. Vercoulen, J. Bourbeau, J. Trappenburg, M.R. Partridge and J. van der Palen), who were also part of the larger expert group. The definition was subsequently modified and emailed to the rest of the expert group as part of the next consensus round. Experts were asked to provide feedback on the proposed definition in each round and score to what extent they agreed with the proposed definition using a five-point Likert scale: $1=$ totally disagree, $2=$ rather disagree, $3=$ neither, $4=$ rather agree, $5=$ totally agree.

The initial definition in round 1 was compiled using the definitions in five literature sources: two recent reviews on COPD self-management $[6,12]$, a landmark study in COPD self-management [10], a recent statement on pulmonary rehabilitation [11], and a previous report of the expert group regarding conformity in COPD self-management [3].

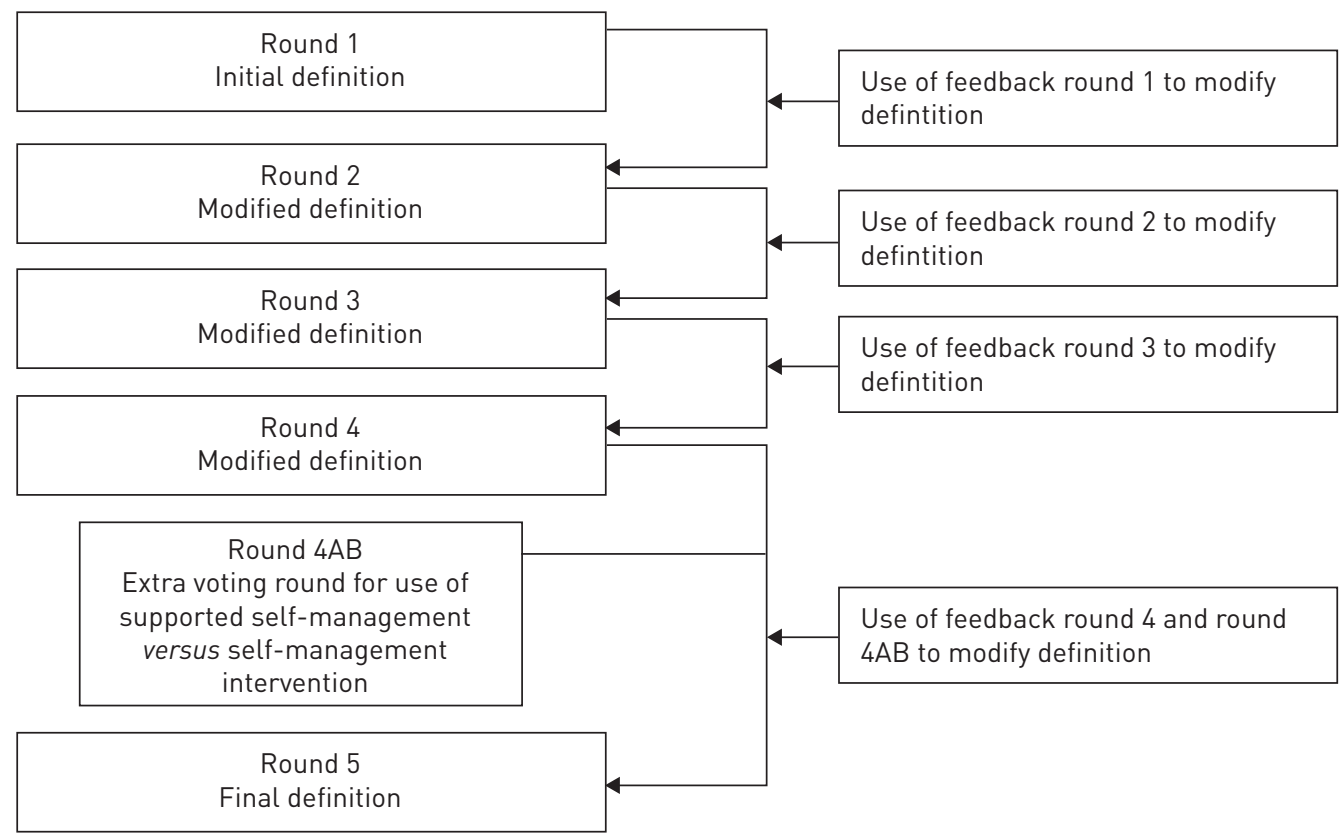

FIGURE 1 Process of developing the definition for a chronic obstructive pulmonary disease self-management intervention. 
The questionnaire used in the first round included an initial definition, questions regarding agreement with the different components of this definition, and questions regarding the sociodemographic status of the expert group members (i.e. age, sex, country of work, occupation, work setting, and years of experience in COPD self-management research/care). In addition, group members were asked to rate their self-perceived scientific skills and clinical expertise regarding COPD on a five-point scale (1=general to $5=$ highly specialised). The questionnaire was pre-tested within the reference group to affirm comprehensibility and was modified accordingly.

In addition to the formal process described above, two extra steps were included in this study. After round 2, 10 expert members developed the definition further by processing the feedback of round 2 during a meeting at the European Respiratory Society International Congress in September 2014. After round 4, the responses indicated that the majority of the group members had very explicit and differing opinions about whether to use the term "supported self-management intervention" or "self-management intervention" within the definition. Therefore, it was determined that an extra voting round (defined as round $4 \mathrm{AB}$ ) should be offered to decide whether to use "supported self-management intervention" or "self-management intervention" in the definition, and it was decided to simply go with a majority of votes.

One expert group member (T.W. Effing) did not contribute to the scores of the five different consensus rounds, but her vote was accounted for in the extra voting round $4 \mathrm{AB}$.

Analyses

Thematic analysis was used for free text responses [16]. Using this method, suggestions for adjustments could be identified and included in the modified definition. In case of doubt, the reference group was consulted and a decision was made on the basis of the majority of votes. Descriptive statistics were used for the agreement scores regarding the definitions in the different rounds, the sociodemographic status data, and the participants' ratings regarding scientific skills and their clinical expertise.

\section{Results}

Expert group members

The characteristics of the 28 expert group members are described in table 1. They were from North America, Europe and Australia, had different professional backgrounds, and the majority worked in both a

\section{TABLE 1 Characteristics of the 28 expert group members}

\section{Characteristics}

\begin{tabular}{lc}
\hline Age years & $51.9 \pm 11.7$ \\
Males & $17(61)$ \\
Country of work & $8(29)$ \\
USA & $2(7)$ \\
Canada & $4(14)$ \\
Australia & $7(25)$ \\
UK & $7(25)$ \\
Netherlands & \\
Occupation & $17(61)$ \\
Medical practitioners & $1(4)$ \\
Nurse & $4(14)$ \\
Physiotherapists & $3(11)$ \\
Psychologist & $8(29)$ \\
Epidemiologist & $10(36)$ \\
Medical researcher & $5(19)$ \\
Work setting" & $1(4)$ \\
$\quad$ Research & $21(78)$ \\
Clinical & $14.5 \pm 7.0$ \\
Research and clinical & $4(3-5)$ \\
Years of experience in COPD self-management research/care & $4(3-5)$ \\
Scientific skills ${ }^{+}$ & \\
Knowledge and/or clinical expertise regarding coPD self-management ${ }^{+}$ & \\
\hline
\end{tabular}

Data are presented as mean \pm SD, $n(\%)$ or median (interquartile range). COPD: chronic obstructive pulmonary disease. \#: multiple answers were possible for each group expert member; ${ }^{\text {ๆ: }} 27$ of 28 group members; ${ }^{+}: 1=$ general, 5=highly specialised. 
clinical and research setting. The members scored their scientific skills with a median (interquartile range) of 4 (3-5) and their knowledge/clinical expertise regarding COPD self-management with a median (interquartile range) of 4 (3-5) on a five-point scale (1=general to $5=$ highly specialised).

\section{Consensus rounds}

The proposed definitions in the five consensus rounds are presented in table 2. The number of responses in each round and the given scores can be found in table 3 . The response rate varied randomly between $48 \%(n=13)$ and $85 \%(n=23)$ over the five rounds and the mean scores increased over the rounds from 3.8 (round 1) to 4.8 (round 5) with an increasing percentage of expert group members allocating the highest score of 5 (round 1: 14\% $(n=3)$; round 5: $83 \%(n=19)$ ).

Experts who favoured the use of "supported self-management intervention" above "self-management intervention" reasoned that the word "supported" distinguished the intervention from a self-help intervention, and that it would clarify the involvement of a healthcare provider/team. Moreover, by omitting "supported" a purely web-based self-management intervention would not meet the definition of a self-management intervention because of the lack of interaction with a healthcare provider. The most important reason given to omit "supported" was that "supporting the patient" is a goal of a self-management intervention and should, therefore, be incorporated in the definition along with other important components. 23 (82\%) out of 28 group members voted in round $4 \mathrm{AB}$ to decide between the terms "supported self-management intervention" and "self-management intervention". 11 group members voted for the use of "supported self-management" and 12 group members for the use of "self-management". As pre-defined, it was decided to adopt the majority vote so the term "self-management" instead of "supported self-management" was used in the fifth and final consensus round.

\section{Open feedback consensus rounds}

Experts were also asked to provide feedback on the proposed definition in rounds 1 to 4 . A broad summary of this information is provided in table 4.

\section{Final definition}

The definition as proposed in round 5 is the final definition for a COPD self-management intervention.

"A COPD self-management intervention is structured but personalised and often multi-component, with goals of motivating, engaging and supporting the patients to positively adapt their health behaviour(s) and develop skills to better manage their disease.

"The ultimate goals of self-management are: a) optimising and preserving physical health; b) reducing symptoms and functional impairments in daily life and increasing emotional well-being, social well-being and quality of life; and c) establishing effective alliances with healthcare professionals, family, friends and community.

"The process requires iterative interactions between patients and healthcare professionals who are competent in delivering self-management interventions. These patient-centred interactions focus on: 1) identifying needs, health beliefs and enhancing intrinsic motivations; 2) eliciting personalised goals; 3) formulating appropriate strategies (e.g. exacerbation management) to achieve these goals; and if required 4) evaluating and re-adjusting strategies. Behaviour change techniques are used to elicit patient motivation, confidence and competence. Literacy sensitive approaches are used to enhance comprehensibility."

\section{Discussion}

For the first time a consensus definition of a COPD self-management intervention was obtained. A high level of agreement on the final definition was achieved by a group of individuals experienced in COPD self-management with a broad international and professional background. $83 \%$ of participants "totally agreeing" with the definition in round 5 is higher than the $70-75 \%$ that is frequently used as a cut-off point for consensus $[14,17]$. Whereas the consensus increased gradually over the study rounds, we needed five study rounds to come to a consensus definition. Theoretically, a Delphi process can be continuously iterated until consensus is reached, but in most Delphi studies three iterations are sufficient to collect the necessary information and reach a consensus [14]. A key contributor to extra study rounds was the controversy around whether or not to use the term "supported self-management" in the definition. An extra voting round was, therefore, initiated after round 4 . Whereas voting was very close, the decision was made to not include the term "supported self-management intervention" in the definition. Including "supported self-management" would have indicated that the intervention was considered a subset of a broader concept of a "self-management intervention". In the final definition, "support" is therefore seen as being intrinsic to a self-management intervention. 
TABLE 2 Proposed definitions in the five rounds of the process

Round Definition

1 (Initial)

Self-management is a term applied to a structured, preferably patient-tailored, multifaceted programme that is directed towards change in health behaviour by training patients and equipping them with skills to both manage and cope with their COPD. The self-management programme requires at least an iterative process of interaction between the patient and healthcare provider that includes: 1) formulation of goals; 2) provision of feedback; 3) training into problem solving and decision making; and 4) training in the use of action plans. Apart from this iterative interaction process, the self-management programme should include at least one of the following components: 1) smoking cessation; 2) self-recognition and/or self-treatment of COPD exacerbations; 3) an exercise or physical activity component; 4) training to cope better with breathlessness; 5) decision making about additional treatment or professional attention; and/or 6) optimising nutrition and/or medication.

A COPD self-management programme is a term applied to a structured, patient-tailored, multifaceted programme in which ongoing support is provided to COPD patients to positively change their health behaviour(s) and to develop skills to better manage their COPD and take more ownership of their own health. This support requires ongoing interaction between the patient and a trained healthcare provider that includes: 1) identifying patient's motivations and needs; 2) eliciting formulation of mutual personalised goals; 3 ) patient training in problem solving and decision making (including the use of action plans and care plans); and 4) provision of feedback by both the healthcare provider and the patient. A COPD self-management programme incorporates behaviour change techniques (including education of appropriate knowledge) aimed at a positive change in behaviour in two of the following groups: 1) preserving or optimising health in "stable" state (e.g. enhancing physical activity, smoking cessation, optimising nutrition, medication, lifestyle, and treatment compliance); 2) self-monitoring of COPD symptoms and initiating appropriate actions when symptoms change (e.g. self-recognition of COPD exacerbations, consulting of healthcare provider(s), self-treatment); 3) assisting the management of the impact of COPD on physical, mental, emotional and social wellbeing (e.g. coping with breathlessness, stress, anxiety, depression and fatigue, energy conservation techniques and engagement in social activities); and 5) improving (assertive) communication with healthcare professionals, family and friends.

A COPD self-management intervention is ongoing, structured, personalised and often multifaceted, with goals of motivating, engaging and supporting the patients to positively change their health behaviour(s) and develop skills to manage their disease more effectively.

This requires iterative interactions of patients with healthcare professionals who are competent in delivering self-management interventions. Interactions should focus on: 1) identifying motivations, needs and health beliefs; 2) eliciting personalised goals; and 3) formulating appropriate strategies (e.g. self-treatment) to achieve these goals.

Behaviour change techniques should be employed to increase motivation to activate and maintain positive COPD management behaviours in order to: 1) optimise and preserve physical health;2) manage the impacts on mental, emotional, and social wellbeing; and 3) establish effective alliances with healthcare professionals, family and friends. Health literacy disparities should be reduced by using literacy sensitive approaches that enhance comprehensibility.

A supported COPD self-management intervention is ongoing, structured but personalised and often multi-component, with goals of motivating, engaging and supporting the patients to positively change their health behaviour(s) and develop skills to better manage their disease.

The ultimate goals of supported self-management are: 1) optimising and preserving physical health; 2) reducing symptoms and functional impairments in daily life and increasing emotional well-being, social well-being and quality of life; and 3) establishing effective alliances with healthcare professionals, family, friends and community.

The process requires iterative interactions of patients with healthcare professionals who are competent in delivering self-management interventions. The interactions focus on: 1) identifying needs, motivations and health beliefs; 2) eliciting personalised goals; 3) formulating appropriate strategies (e.g. exacerbation management) to achieve these goals; and 4) if required, evaluating and re-adjusting strategies. Behaviour change techniques are used to elicit patient motivation, confidence and competence. Literacy sensitive approaches are used to enhance comprehensibility.

5 (Final)

A COPD self-management intervention is structured but personalised and often multi-component, with goals of motivating, engaging and supporting the patients to positively adapt their health behaviour(s) and develop skills to better manage their disease.

The ultimate goals of self-management are: al optimising and preserving physical health; b) reducing symptoms and functional impairments in daily life and increasing emotional well-being, social well-being and quality of life; and c) establishing effective alliances with healthcare professionals, family, friends and community.

The process requires iterative interactions between patients and healthcare professionals who are competent in delivering self-management interventions. These patient-centred interactions focus on: 1) identifying needs, health beliefs and enhancing intrinsic motivations; 2) eliciting personalised goals; 3) formulating appropriate strategies (e.g. exacerbation management) to achieve these goals; and if required 4) evaluating and re-adjusting strategies. Behaviour change techniques are used to elicit patient motivation, confidence and competence. Literacy sensitive approaches are used to enhance comprehensibility.

COPD: chronic obstructive pulmonary disease. 
TABLE 3 Results from the five consensus rounds ${ }^{\#}$

\begin{tabular}{lccc} 
Round & Responses & Score & Responses with a score of $\mathbf{5}^{\boldsymbol{\pi}}$ \\
\hline $\mathbf{1}$ (Initial) & $21(78)$ & $3.8 \pm 0.87$ & $3(14)$ \\
$\mathbf{2}$ & $14(52)$ & $4.2 \pm 0.58$ & $4(29)$ \\
$\mathbf{3}$ & $13(48)$ & $4.5 \pm 0.52$ & $6(46)$ \\
$\mathbf{4}$ & $21(78)$ & $4.6 \pm 0.50$ & $13(62)$ \\
$\mathbf{5}$ (Final) & $23(85)$ & $4.8 \pm 0.39$ & $19(83)$
\end{tabular}

Data are presented as $\mathrm{n}(\%)$ or mean $\pm \mathrm{SD}$. \# : maximum possible number of responses is 27 because one expert member did not contribute to these scores ratings; ": five-point Likert scale: 1=totally disagree, 2=rather disagree, 3=neither, 4=rather agree, 5=totally agree.

A clear modification of the COPD self-management intervention definition can be observed during the consensus process. First, COPD self-management intervention goals and the focus points for patient-centred interactions have been defined from round 4 onwards. Secondly, the description of the specific intervention components included in the first rounds were removed because it was agreed that the aim of the study was not to come to an operational definition, but to define the concept of COPD self-management. Another contrast with the initial definition is the emphasis given to intrinsic motivations, behaviour change techniques and literacy sensitive approaches in the final definition. Finally, a novel aspect of the final definition is accentuating the "right affect" in the patient at the time of exposure to the COPD self-management intervention (i.e. "engaging, and supporting the patients to positively adapt their health behaviour"). Recent data claim that the positivity of the patient is a critical factor for a self-management intervention to increase wellbeing in COPD [18].

Motivation is a crucial but rather neglected concept in COPD management in general [19]. Besides developing skills, enhancing the patient's motivation to actually execute these skills on a daily basis is an essential component of a COPD self-management intervention. Far too often interventions are stopped when the healthcare provider concludes that the patient is not motivated [4]. A client-centred communication style is essential to elicit intrinsic motivation in the patient, one of the most critical components to enable behaviour change [20,21]. Motivational interviewing is one technique that can enable goal formulation and ensure that goals are personally meaningful to the patient, which is crucial to enhance intrinsic motivation [4, 22]. For behaviour change to occur, patients need to feel that it will benefit them personally and that change is achievable. Behaviour change is more likely if the new behaviours lead to personally relevant and valued outcomes.

Successful self-management involves the ability to adapt one's behaviour over time according to changing circumstances (e.g. sudden symptom increases associated with exacerbations, alterations in social network

\section{TABLE 4 Broad summary of feedback from rounds 1 to 4}

\section{Discussion feedback round 1}

Exact wording, terminology must be instantly understandable, addition of further explanations in parentheses

"Mandatory" components of a self-management intervention, grouping and re-organising of these components, and rationale why

Difference between self-management versus self-management support/intervention/programme

\section{Discussion feedback round 2}

Shortening of definition

Role of motivation

Distinction between ultimate treatment goals and the interventions by which we achieve these goals

\section{Discussion feedback round 3}

Goal of behaviour change is to facilitate confidence and competence as well as motivation

Should "ongoing" be included and statement for the need for an adequate assessment?

Addition of "evaluations of strategies"

Does definition need to be modified so it can be used by commissioners to purchase services?

Difference with self-care

Supported self-management versus self-management

Discussion feedback round 4

Removal of term "ongoing" and question whether behaviour should be "permanently" changed/adapted

Inclusion of patient centred

Supported self-management versus self-management 
and changing status of disease). Behavioural change evolves over time, with different patterns and timelines for different people. Health behaviours that are preferred at one point may not be needed at a later point in time. Sedentary persons would, for example, need to make a permanent change to their physical activity status, but would still need to be able to adapt and vary the type and level of their physical activities or exercises depending on circumstances. Therefore, it was decided to avoid the use of the term "permanent" in the definition. One of the permanent changes that should be a prime goal, however, is increasing the patient's ability to adapt.

Besides the pathophysiological causes of COPD, poor self-management behaviours are believed to have a significant influence on symptoms, functional impairments and quality of life [6]. This is reflected in the defined goals of a self-management intervention. Healthy behaviours that already exist should, of course, be preserved and strengthened. The individual patient's needs, preferences and personal goals should therefore inform the tailoring of any self-management intervention.

We envisage the development of a conceptual definition as a necessary and important first step towards an operational definition of a COPD self-management intervention. It clarifies the boundaries of what should and should not be considered as a COPD self-management intervention and so facilitates the development, implementation and evaluation of future interventions. Having this conceptual definition also specifies the position of the intervention that is frequently embedded in other therapeutic entities such as pulmonary rehabilitation and integrated care. The fast growing body of evidence regarding COPD self-management interventions will provide us with further opportunities to operationalise this conceptual definition.

Our study includes some potential limitations. Whereas our methods include many features of a Delphi technique (e.g. iterative process, participants can adjust initial ratings based on group feedback, and individual scoring), it cannot be viewed as a classic Delphi study [14]. The fact that the participants knew each other and not all communication was anonymised deviates from a classic Delphi technique [23]. In addition, features of other consensus techniques such as "consensus conferences" [15] were included during an organised group meeting half way through the consensus process. Another possible limitation of this study may be the group composition [1]. Most group members had known one another for a long period of time because the field of self-management is still relatively small. Therefore, it is expected that thoughts and beliefs of some participants may have influenced other participants. However, we feel that it is not necessarily a negative aspect while striving for more agreement in the field. All participants were considered as experts in COPD self-management with most having contributed significantly to the research published in past decades and we believe that they have formed and expressed an opinion that reflects their own experience and expertise. Another limitation is the fact that only one nurse was involved in this consensus process. Nurses have a very important role within COPD self-management programmes and the number of participating nurses should ideally have been higher. Besides more nurses we will also consider including patients in future processes.

\section{Conclusions and recommendation}

In this study we reached consensus regarding a conceptual definition of what should be a COPD self-management intervention, clarifying the requisites for such an intervention. Operationalisation of this conceptual definition in the near future will be an essential next step.

\section{References}

1 Lorig KR, Holman H. Self-management education: history, definition, outcomes, and mechanisms. Ann Behav Med 2003; 26: 1-7.

2 Barlow J, Wright C, Sheasby J, et al. Self-management approaches for people with chronic conditions: a review. Patient Educ Couns 2002; 48: 177-187.

3 Effing TW, Bourbeau J, Vercoulen J, et al. Self-management programmes for COPD: moving forward. Chron Respir Dis 2012; 9: 27-35.

4 Rollnick S, Miller WR, Butler CC. Motivational Interviewing in Health Care: Helping Patients Change Behavior. New York, Guilford Press, 2008.

5 Global Initiative for Chronic Obstructive Lung Disease. Global strategy for the diagnosis, management and prevention of chronic obstructive lung disease (GOLD) 2016. www.goldcopd.org Date last accessed: March 29, 2016.

6 Zwerink M, Brusse-Keizer M, van der Valk PD, et al. Self-management for patients with chronic obstructive pulmonary disease. Cochrane Database Syst Rev 2014; 3: CD002990.

7 Leiva-Fernandez J, Leiva-Fernandez F, Vazquez-Alarcon RL, et al. Study protocol for a randomized, controlled trial comparing the efficacy of two educational interventions to improve inhalation techniques in patients with chronic obstructive pulmonary disease (COPD): TIEPOC Study. Drugs Context 2014; 3: 212261.

8 Effing T, Kerstjens $\mathrm{H}$, van der Valk $\mathrm{P}$, et al. (Cost)-effectiveness of self-treatment of exacerbations on the severity of exacerbations in patients with COPD: the COPE II study. Thorax 2009; 64: 956-962.

9 van Wetering CR, Hoogendoorn M, Mol SJ, et al. Short- and long-term efficacy of a community-based COPD management programme in less advanced COPD: a randomised controlled trial. Thorax 2010; 65: 7-13.

10 Bourbeau J, Julien M, Maltais F, et al. Reduction of hospital utilization in patients with chronic obstructive pulmonary disease: a disease-specific self-management intervention. Arch Intern Med 2003; 163: 585-591. 
11 Spruit MA, Singh SJ, Garvey C, et al. An official American Thoracic Society/European Respiratory Society statement: key concepts and advances in pulmonary rehabilitation. Am J Respir Crit Care Med 2013; 188: e13-e64.

12 Jonkman NH, Westland $\mathrm{H}$, Trappenburg JC, et al. Towards tailoring of self-management for patients with chronic heart failure or chronic obstructive pulmonary disease: a protocol for an individual patient data meta-analysis. BMJ Open 2014; 4: e005220.

13 Wagg K. Unravelling self-management for COPD: what next? Chron Respir Dis 2012; 9: 5-7.

14 Hsu CC, Sandford BA. The Delphi technique: making sense of consensus. Practical Assess Res Eval 2007; 12: 1-8.

15 Nielsen AP, Hansen J, Skorupinski B, et al. Consensus conference: manual. The Hague, Agricultural Economics Research Institute (LEI), 2006.

16 Braun V, Clarke V. Using thematic analysis in psychology. Qual Res Psychol 2006; 3: 77-101.

17 Fitch K, Bernstein SJ, Aguilar MD, et al. The RAND/UCLA Appropriateness Method User's Manual. Arlington, RAND Corporation, 2001.

18 Benzo RP, Abascal-Bolado B, Dulohery MM. Self-management and quality of life in chronic obstructive pulmonary disease (COPD): the mediating effects of positive affect. Patient Educ Couns 2016; 99: 617-623.

19 Vercoulen JH. A simple method to enable patient-tailored treatment and to motivate the patient to change behaviour. Chron Respir Dis 2012; 9: 259-268.

20 Dacey M, Baltzell A, Zaichkowsky L. Older adults' intrinsic and extrinsic motivation toward physical activity. Am J Health Behav 2008; 32: 570-582.

21 Furia AC, Lee RE, Strother ML, et al. College students' motivation to achieve and maintain a healthy weight. Am J Health Behav 2009; 33: 256-263.

22 Benzo R, Vickers K, Ernst D, et al. Development and feasibility of a self-management intervention for chronic obstructive pulmonary disease delivered with motivational interviewing strategies. J Cardiopulm Rehabil Prev 2013; 33: 113-123.

23 Diamond IR, Grant RC, Feldman BM, et al. Defining consensus: a systematic review recommends methodologic criteria for reporting of Delphi studies. J Clin Epidemiol 2014; 67: 401-409. 